\title{
Existence and uniqueness of solutions for certain functional equations and system of functional equations arising in dynamic programming
}

\author{
Deepmala, Ravi P. Agarwal
}

\begin{abstract}
This paper deals with the existence, uniqueness and iterative approximations of solutions for the functional equations and system of functional equations arising in dynamic programming of multistage decision making processes in Banach spaces and complete metric space, respectively. The results presented in this paper unify and generalize many known results in the literature. Some examples which dwell upon the importance of our results are also illustrated.
\end{abstract}

\section{Introduction}

Bellman [1, 2] introduced and studied the existence of solutions for a class of functional equations arising in dynamic programming. Since then many authors (see, [3] - [15]) have established the existence and uniqueness of solutions of functional equations by modifying the conditions of Bellman equations arising in dynamic programming. In this paper, we introduce and study the existence and uniqueness of solutions for the following functional equations and

Key Words: Dynamic programming, Functional equations, Fixed point

2010 Mathematics Subject Classification: Primary 49L20, 49L99, 90C39; Secondary 47A30, 47B47.

Received: July, 2015.

Accepted: September, 2015. 
EXISTENCE AND UNIQUENESS OF SOLUTIONS FOR CERTAIN FUNCTIONAL EQUATIONS AND SYSTEM OF FUNCTIONAL EQUATIONS ARISING IN DYNAMIC PROGRAMMING

system of functional equations arising in dynamic programming of multistage decision processes:

$$
\begin{aligned}
f(x) & =o_{y} t_{y \in D}\left\{u(x, y)+o p t\left\{v(x, y), p_{i}(x, y)+A_{i}\left(x, y, f\left(a_{i}(x, y)\right)\right): i=1,2,3\right\}\right\} \\
f(x) & =o_{t} t_{y \in D}\{u(x, y)+r(x, y) f(c(x, y)) \\
& \left.+o p t\left\{v(x, y), p(x, y) f(s(x, y)), t_{i}(x, y)+q_{i}(x, y) f\left(a_{i}(x, y)\right): i=1,2,3\right\}\right\}
\end{aligned}
$$

and

$$
\begin{aligned}
& f(x)=\operatorname{opt}_{y \in D}\left\{p(x, y)+o p t\left\{u_{i}(x, y)+A_{i}\left(x, y, g\left(a_{i}(x, y)\right)\right): i=1,2,3\right\}\right\} \\
& g(x)=\operatorname{opt}_{y \in D}\left\{q(x, y)+o p t\left\{v_{i}(x, y)+B_{i}\left(x, y, f\left(b_{i}(x, y)\right)\right): i=1,2,3\right\}\right\}
\end{aligned}
$$

where "opt" denotes the "sup" or "inf", $x$ and $y$ stand for the state and decision vectors respectively, $s, c$ and $a_{i}, i=1,2,3$ represent the transformation of the processes, $f(x)$ and $g(x)$ denote the optimal return function with initial state $x$.

We note that (1), (2) and (3) include many functional equations and system of functional equations as special cases. For example, the functional equations

$$
\begin{aligned}
& f(x)=\inf _{y \in D} \max \{r(x, y), s(x, y), f(b(x, y))\}, \quad x \in S . \\
& f(x)=\inf _{y \in D} \max \{r(x, y), f(b(x, y))\}, \quad x \in S . \\
& f(x)=\text { opt }_{y \in D} \max \{u(x, y), f(T(x, y))\}, \quad x \in S . \\
& f(x)=\sup _{y \in D}\{p(x, y)+A(x, y, f(a(x, y)))\}, \quad x \in S . \\
& f(x)=\sup _{y \in D}\{p(x, y)+f(a(x, y))\}, \quad x \in S . \\
& f(x)=o p t_{y \in D}\{a[u(x, y)+f(T(x, y))]+b \text { opt }[v(x, y), f(T(x, y))]\}, \\
& x \in S, a+b=1 . \\
& f(x)=\operatorname{opt}_{y \in D}\left\{u(x, y)+o p t\left\{p_{i}(x, y)+q_{i}(x, y) f_{i}\left(a_{i}(x, y)\right): i=1,2\right\}\right\} \text {, } \\
& x \in S \text {. } \\
& f(x)=o p t_{y \in D}\{p(x, y)+q(x, y) f(a(x, y))+o p t\{r(x, y), s(x, y) f(b(x, y)) \text {, } \\
& t(x, y) f(c(x, y))\}\}, \forall x \in S \text {. } \\
& f(x)=\inf _{y \in D} \max \{p(x, y), f(a(x, y)), q(x, y)+f(b(x, y))\}, \forall x \in S . \\
& f(x)=o p t_{y \in D} \operatorname{opt}\{p(x, y), q(x, y) f(a(x, y)), r(x, y) f(b(x, y)), s(x, y) f(c(x, y))\} \\
& f(x)=\text { opt }_{y \in D}\{p(x, y)+A(x, y) f(a(x, y))+ \\
& \operatorname{opt}\{q(x, y)+B(x, y) f(b(x, y)), r(x, y)+C(x, y) f(c(x, y))\}\} .
\end{aligned}
$$


These equations have been studied by Bellman [2], Bhakta and Mitra [3], Bhakta and Chuodhary [4], Liu and Ume [11], Liu et al. [12, 16], Liu and Kang [10], Jiang et al. [8], Liu [9], Liu et al. [14], Liu et al. [13], and Pathak and Deepmala [17], respectively. They established the existence of solutions of functional equations (4)-(15) in the spaces $B C(S), B(S)$ or $B B(S)$ (defined in Section 2), respectively. The systems of functional equations

$$
\begin{gathered}
f(x)=\sup _{y \in D}\{u(x, y)+G(x, y, g(a(x, y)))\} \\
g(x)=\sup _{y \in D}\{u(x, y)+F(x, y, f(a(x, y)))\} \\
f(x)=\sup _{y \in D}\{v(x, y)+G(x, y, g(a(x, y)))\} \\
g(x)=\sup _{y \in D}\{u(x, y)+F(x, y, f(b(x, y)))\} \\
f(x)=\sup _{y \in D} \operatorname{opt}\{p(x, y), A(x, y, g(a(x, y))), q(x, y)+B(x, y, g(b(x, y)))\}, \forall x \in S, \\
g(x)=\sup _{y \in D} \operatorname{opt}\{u(x, y), C(x, y, g(a(x, y))), v(x, y)+H(x, y, f(h(x, y)))\}, \forall x \in S,
\end{gathered}
$$

have been studied by Chang [6], Chang and Ma [7] and Liu et al. [15].

The plan of our paper is as follows: In Section 2, we recall some basic concepts, notations, and Lemmas. In Section 3, we apply the fixed point theorem of Boyd and Wong [5] to establish the existence, uniqueness, and iterative approximation of solutions for the generalized type of system of functional equations (3) in $B B(S)$. In Section 4, we establish the existence, uniqueness, and iterative approximations of solutions of functional equation (1) in $B C(S)$ and $B(S)$. In Section 5, we obtain the existence, uniqueness, and iterative approximations of solutions of functional equation (2) in $B B(S)$. To show how our results can be used in practice we also illustrate some nontrivial examples. The results presented here generalize and unify the results of Bellman [1], Bhakta and Mitra [3], Bhakta and Choudhury [4], Liu and Ume [11], Liu et al. [12], Liu et al. [16], Liu and Kang [10], Jiang et al. [8], Liu [9], Liu et al. [14], Liu et al. [13], Pathak and Deepmala [17] Chang [6], Chang and Ma [7], and Liu et al. [15].

\section{Preliminaries}

In this section, we introduce notations, definitions, and some results that will be used in the remainder of the paper. Let $\mathbb{R}=(-\infty,+\infty), R^{+}=[0,+\infty)$ and $R^{-}=(-\infty, 0]$. For any $t \in \mathbb{R},[t]$ denotes the largest integer not exceeding 
$t$ and $(X,\|\|$.$) and \left(Y,\|.\|^{\prime}\right)$ be real Banach spaces. $S \subseteq X$ be the state space and $D \subseteq Y$ be the decision space. Define

$\Phi_{1}=\left\{\varphi \mid \varphi: R^{+} \rightarrow R^{+}\right.$is right continuous at $\left.t=0\right\}$,

$\Phi_{2}=\left\{\varphi \mid \varphi: R^{+} \rightarrow R^{+}\right.$is nondecreasing $\}$,

$\Phi_{3}=\left\{\varphi \mid \varphi \in \Phi_{1}\right.$ and $\left.\varphi(0)=0\right\}$,

$\Phi_{4}=\left\{\varphi \mid \varphi \in \Phi_{1} \cap \Phi_{2}\right.$ and $\varphi(t)<t$ for $\left.t>0\right\}$

$\Phi_{5}=\left\{(\varphi, \psi) \mid \varphi\right.$ and $\psi: R^{+} \rightarrow R^{+}$are nondecreasing and $\sum_{n=0}^{\infty} \psi\left(\varphi^{n}(t)\right)<$ $\infty$ for $t>0\}$,

$\Phi_{6}=\left\{(\varphi, \psi) \mid(\varphi, \psi) \in \Phi_{5}\right.$ and $\left.\psi(t)>0, \forall t>0\right\}$,

$\Phi_{7}=\left\{\varphi \mid \varphi \in \Phi_{2}\right.$ and $\varphi(t)<t$ for $\left.t>0\right\}$,

$\Phi_{8}=\left\{(\varphi, \psi) \mid \varphi\right.$ and $\psi: R^{+} \rightarrow R^{+}$are nondecreasing $\psi(t)>0$ and $\lim _{n \rightarrow \infty} \psi\left(\varphi^{n}(t)\right)=0$ for $\left.t>0\right\}$,

$\Phi_{9}=\left\{\varphi \mid \varphi: R^{+} \rightarrow R^{+}\right.$is nondecreasing and $\sum_{n=0}^{\infty} \varphi^{n}(t)<\infty$ for $\left.t>0\right\}$, $B(S)=\{f \mid f: S \longrightarrow \mathbb{R}$ is bounded $\}$,

$B C(S)=\{f \mid f \in B(S)$ is continuous $\}$,

$B B(S)=\{f \mid f: S \longrightarrow \mathbb{R}$ is bounded on bounded subsets of $\mathrm{S}\}$.

Clearly, $\left(B(S),\|\cdot\|_{1}\right)$ and $\left(B C(S),\|\cdot\|_{1}\right)$ are Banach spaces with the norm $\|f\|_{1}=\sup _{x \in S}|f(x)|$. For any positive integer $k$ and $f, g \in B B(S)$, let

$$
\begin{gathered}
d_{k}(f, g)=\sup \{|f(x)-g(x)|: x \in \bar{B}(0, k)\} \\
d(f, g)=\sum_{k=1}^{\infty} \frac{1}{2^{k}} \cdot \frac{d_{k}(f, g)}{1+d_{k}(f, g)}
\end{gathered}
$$

where $\bar{B}(0, k)=\{x: x \in S$ and $\|x\| \leq k\}$. Then $\left\{d_{k}\right\}_{k \geq 1}$ is a countable family of pseudometrices on $B B(S)$. A sequence $\left\{x_{n}\right\}_{n \geq 1}$ in $B B(S)$ is said to converge to a point $x \in B B(S)$ if for any $k \geq 1, \bar{d}_{k}\left(x_{n}, x_{m}\right) \longrightarrow 0$ as $n, m \longrightarrow \infty$. It is clear that $(B B(S), d)$ is a complete metric space. A metric space $(M, \rho)$ is said to be metrically convex if for each $x, y \in M$, there is a $z \neq x, y$ for which $\rho(x, y)=\rho(x, z)+\rho(z, y)$. Clearly, any Banach space is metrically convex.

Lemma 2.1. [5] Suppose that $(M, \rho)$ is a completely metrically convex metric space and $f: M \longrightarrow M$ satisfies

$$
\rho(f(x), f(y)) \leq \varphi(\rho(x, y)) \quad \text { for } x, y \in M,
$$

where $\varphi: \bar{P} \longrightarrow R^{+}$satisfies $\varphi(t)<t$ for $t \in \bar{P}-\{0\}$, where $P=\{\rho(x, y)$ : $x, y \in M\}$ and $\bar{P}$ denotes the closure of $P$. Then $f$ has a fixed point $u \in M$ and $\lim _{n \longrightarrow \infty} f^{n}(x)=u$ for each $x \in M$.

Lemma 2.2. $[8]$ Let $\left\{a_{i}, b_{i}: 1 \leq i \leq n\right\} \subseteq \mathbb{R}$. Then

$$
\left|\operatorname{opt}\left\{a_{i}: 1 \leq i \leq n\right\}-\operatorname{opt}\left\{b_{i}: 1 \leq i \leq n\right\}\right| \leq \max \left\{\left|a_{i}-b_{i}\right|: 1 \leq i \leq n\right\} .
$$


Lemma 2.3. [8] $(i)$ Assume that $A: S \times D \rightarrow \mathbb{R}$ is a mapping such that opt $_{y \in D} A\left(x_{0}, y\right)$ is bounded for some $x_{0} \in S$. Then

$$
\mid \text { opt }_{y \in D} A\left(x_{0}, y\right)\left|\leq \sup _{y \in D}\right| A\left(x_{0}, y\right) \mid \text {; }
$$

(ii) Assume that $A, B: S \times D \rightarrow \mathbb{R}$ is a mapping such that opt $\operatorname{ta}_{y} A\left(x_{1}, y\right)$ and opt ${ }_{y \in D} B\left(x_{2}, y\right)$ are bounded for some $x_{1}, x_{2} \in S$. Then

$$
\mid \text { opt }_{y \in D} A\left(x_{1}, y\right)-\text { opt }_{y \in D} B\left(x_{2}, y\right)\left|\leq \sup _{y \in D}\right| A\left(x_{1}, y\right)-B\left(x_{2}, y\right) \mid .
$$

To prove our results we also need the following lemma:

Lemma 2.4. Let $a_{i}, b_{i}, i=1,2,3$ be in $\mathbb{R}$. Then

$$
\max \left\{\left|a_{i}+b_{i}\right|: i=1,2,3\right\} \leq \max \left\{\left|a_{i}\right|: i=1,2,3\right\}+\max \left\{\left|b_{i}\right|: i=1,2,3\right\}
$$

Proof. The proof of this lemma is based on some geometrical properties.

Algorithm 1. For any $f_{0} \in B B(S)$, compute $\left\{f_{n}\right\}_{n \geq 0}$ by

$$
\begin{aligned}
& f_{n+1}(x)=\left(1-\alpha_{n}\right) f_{n}(x)+\alpha_{n} \text { opt }_{y \in D}\left\{u(x, y)+r(x, y) f_{n}(c(x, y))\right. \\
& \left.\quad+\operatorname{opt}\left\{v(x, y), p(x, y) f_{n}(s(x, y)), t_{i}(x, y)+q_{i}(x, y) f_{n}\left(a_{i}(x, y)\right): i=1,2,3\right\}\right\}, \\
& \quad \forall x \in S, n \geq 0,
\end{aligned}
$$

where

$$
\left\{\alpha_{n}\right\}_{n \geq 0} \text { is any sequence in }[0,1] \text { such that } \sum_{n=0}^{\infty} \alpha_{n}=+\infty \text {. }
$$

Algorithm 2. For any $w_{0} \in B B(S)$, compute $\left\{w_{n}\right\}_{n \geq 0}$ by

$$
\begin{aligned}
& w_{n+1}(x)=\operatorname{opt}_{y \in D}\left\{u(x, y)+r(x, y) w_{n}(c(x, y))\right. \\
& \left.+\operatorname{opt}\left\{v(x, y), p(x, y) w_{n}(s(x, y)), t_{i}(x, y)+q_{i}(x, y) w_{n}\left(a_{i}(x, y)\right): i=1,2,3\right\}\right\}, \\
& \forall x \in S, n \geq 0,
\end{aligned}
$$

\section{$3 \quad$ Existence of coincidence solutions for system of functional equations}

$\bar{f}$ and $\bar{g}$ are said to be coincidence solutions of the system of functional equations (3) if they satisfy the following condition

$$
\begin{aligned}
& \bar{f}(x)=\text { opt }_{y \in D}\left\{p(x, y)+\operatorname{opt}\left\{u_{i}(x, y)+A_{i}\left(x, y, \bar{g}\left(a_{i}(x, y)\right)\right): i=1,2,3\right\}\right\} \\
& \bar{g}(x)=\text { opt }_{y \in D}\left\{q(x, y)+o p t\left\{v_{i}(x, y)+B_{i}\left(x, y, \bar{f}\left(b_{i}(x, y)\right)\right): i=1,2,3\right\}\right\} .
\end{aligned}
$$


Theorem 3.1. Let $a_{i}, b_{i}: S \times D \rightarrow S, u_{i}, v_{i}: S \times D \rightarrow \mathbb{R}$ and $A_{i}, B_{i}:$ $S \times D \times \mathbb{R} \rightarrow \mathbb{R}$ for $i=1,2,3$, and $(\varphi, \psi)$ be in $\Phi_{5}$ satisfying the following conditions:

$\left(D_{1}\right) \max \left\{|p(x, y)|+\max \left\{\left|u_{i}(x, y)\right|: i=1,2,3\right\},|q(x, y)|+\max \left\{\left|v_{i}(x, y)\right|:\right.\right.$ $i=1,2,3\}\} \leq \psi(\|x\|)$, for all $(x, y) \in S \times D$,

$\left(D_{2}\right) \max \left\{\left\|a_{i}(x, y)\right\|,\left\|b_{i}(x, y)\right\|: i=1,2,3\right\} \leq \varphi(\|x\|)$, for all $(x, y) \in S \times D$,

$\left(D_{3}\right)$ opt $\left\{v_{i}(x, y)+B_{i}(x, y, z): i=1,2,3\right\} \geq 0$ and $\max \left\{\left|A_{i}(x, y, z)\right|,\left|B_{i}(x, y, z)\right|: i=1,2,3\right\} \leq|z|, \quad$ for all $(x, y, z) \in$ $S \times D \times \mathbb{R}$

$\left(D_{4}\right)$ for $(x, y) \in S \times D, A_{i}(x, y,$.$) and B_{i}(x, y,$.$) are both left continuous and$ nondecreasing with respect to the third argument on $\mathbb{R}$ for $i=1,2,3$.

Then the system of functional equations

$$
\begin{aligned}
& f(x)=\sup _{y \in D}\left\{p(x, y)+o p t\left\{u_{i}(x, y)+A_{i}\left(x, y, g\left(a_{i}(x, y)\right)\right): i=1,2,3\right\}\right\} \\
& g(x)=\sup _{y \in D}\left\{q(x, y)+\operatorname{opt}\left\{v_{i}(x, y)+B_{i}\left(x, y, f\left(b_{i}(x, y)\right)\right): i=1,2,3\right\}\right\}
\end{aligned}
$$

possesses coincidence solutions $f$ and $g$ in $B B(S)$.

Proof. Let

$$
\begin{gathered}
g_{0}(x)=\sup _{y \in D} q(x, y) \forall x \in S, \\
g_{2 n}(x)=\sup _{x \in D}\left\{q(x, y)+o p t\left\{v_{i}(x, y)+B_{i}\left(x, y, f_{2 n-1}\left(b_{i}(x, y)\right): i=1,2,3\right)\right\}\right\}, \\
n \geq 1,
\end{gathered}
$$

and

$$
\begin{aligned}
& f_{2 n+1}(x)=\sup _{y \in D}\left\{p(x, y)+o p t\left\{u_{i}(x, y)+A_{i}\left(x, y, g_{2 n}\left(a_{i}(x, y)\right): i=1,2,3\right)\right\}\right\} \\
& n \geq 0 .
\end{aligned}
$$

It follows from $\left(D_{4}\right)$ that for any $x \in S$,

$$
\begin{gathered}
g_{0}(x) \leq g_{2}(x) \leq \ldots \leq g_{2 n}(x) \leq g_{2 n+2}(x) \leq \ldots \\
f_{1}(x) \leq f_{3}(x) \leq \ldots \leq f_{2 n-1}(x) \leq f_{2 n+1}(x) \leq \ldots .
\end{gathered}
$$


Let $x \in S$ and $k$ be a positive integer with $x \in \bar{B}(0, k)$. Then by Lemma 2.2, Lemma 2.3 , and $\left(D_{1}\right)$, we find

$$
\left|g_{0}(x)\right|=\left|\sup _{y \in D} q(x, y)\right| \leq \psi(\| x||)
$$

According to Lemma 2.4 and $\left(D_{1}\right)-\left(D_{3}\right)$, we have

$$
\begin{aligned}
& \left|f_{1}(x)\right| \\
& =\mid \sup _{y \in D}\left\{p(x, y)+\operatorname{opt}\left\{u_{i}(x, y)+A_{i}\left(x, y, g_{0}\left(a_{i}(x, y)\right): i=1,2,3\right\}\right\} \mid\right. \\
& \leq \sup _{y \in D}\left\{|p(x, y)|+\max \left\{\left|u_{i}(x, y)+A_{i}\left(x, y, g_{0}\left(a_{i}(x, y)\right)\right)\right|: i=1,2,3\right\}\right\} \\
& \leq \sup _{y \in D}\left\{|p(x, y)|+\max \left\{\left|u_{i}(x, y)\right|: i=1,2,3\right\}\right. \\
& \left.+\max \left\{\left|A_{i}\left(x, y, g_{0}\left(a_{i}(x, y)\right)\right)\right|: i=1,2,3\right\}\right\} \\
& \leq \sum_{i=0}^{1} \psi\left(\varphi^{i}(\|x\|)\right)
\end{aligned}
$$

By using a similar argument, we obtain

$$
\begin{gathered}
\left|g_{2 n}(x)\right| \leq \sum_{i=0}^{2 n} \psi\left(\varphi^{i}(\|x\|)\right) \leq \sum_{i=0}^{\infty} \psi\left(\varphi^{i}(k)\right), \quad \forall n \geq 0, \\
\left|f_{2 n+1}(x)\right| \leq \sum_{i=0}^{2 n+1} \psi\left(\varphi^{i}(\|x\|)\right) \leq \sum_{i=0}^{\infty} \psi\left(\varphi^{i}(k)\right), \quad \forall n \geq 0 .
\end{gathered}
$$

Thus (31) and (32) ensure that $\left\{g_{2 n}(x)\right\}_{n \geq 0}$ and $\left\{f_{2 n+1}(x)\right\}_{n \geq 0}$ are both bounded.

By virtue of (28) - (32), we conclude that

$$
\lim _{n \rightarrow \infty} g_{2 n}(x)=g(x), \lim _{n \rightarrow \infty} f_{2 n+1}(x)=f(x), \forall x \in \bar{B}(0, k),
$$

and

$$
\max \{|f(x)|,|g(x)|\} \leq \sum_{i=0}^{\infty} \psi\left(\varphi^{i}(k)\right), \quad \forall x \in \bar{B}(0, k) .
$$

It is easy to see that (34) yields that $f, g \in B B(S)$. Set

$$
\begin{aligned}
& M(x)=\sup _{y \in D}\left\{p(x, y)+\operatorname{opt}\left\{u_{i}(x, y)+A_{i}\left(x, y, g\left(a_{i}(x, y)\right)\right): i=1,2,3\right\}\right\}, x \in S, \\
& N(x)=\sup _{y \in D}\left\{q(x, y)+\operatorname{opt}\left\{v_{i}(x, y)+B_{i}\left(x, y, f\left(b_{i}(x, y)\right)\right): i=1,2,3\right\}\right\}, x \in S .
\end{aligned}
$$


Notice that (25) - (27) and (35) imply that for any $(x, y) \in S \times D$,

$$
\begin{aligned}
& p(x, y)+o p t\left\{u_{i}(x, y)+A_{i}\left(x, y, g_{2 n}\left(a_{i}(x, y)\right): i=1,2,3\right)\right\} \leq f_{2 n+1}(x) \leq M(x), \\
& q(x, y)+o p t\left\{v_{i}(x, y)+B_{i}\left(x, y, f_{2 n-1}\left(b_{i}(x, y)\right): i=1,2,3\right)\right\} \leq g_{2 n}(x) \leq N(x) .
\end{aligned}
$$

Letting $n \rightarrow \infty$ in $(36),\left(D_{4}\right),(28)-(29)$ and (33), we find

$$
\begin{aligned}
& p(x, y)+o p t\left\{u_{i}(x, y)+A_{i}\left(x, y, g\left(a_{i}(x, y)\right): i=1,2,3\right)\right\} \leq f(x) \leq M(x) \\
& q(x, y)+o p t\left\{v_{i}(x, y)+B_{i}\left(x, y, f\left(b_{i}(x, y)\right): i=1,2,3\right)\right\} \leq g(x) \leq N(x)
\end{aligned}
$$

Which yields that

$$
\begin{aligned}
& M(x) \leq f(x) \leq M(x), \\
& N(x) \leq g(x) \leq N(x) .
\end{aligned}
$$

That is, $M(x)=f(x)$ and $N(x)=g(x)$, for $x \in S$. Therefore, $f$ and $g$ are coincidence solutions of the system of functional equations (24). This completes the proof.

Remark 3.2. 1. It we put $u_{i}=v_{i}=0$ for $i=1,2,3$, then Theorem 3.1 reduces to Theorem 3.1 of Liu et al. [12].

2. Theorem 3.2 and Theorem 3.3 of Liu et al. [12] and Theorem 4.1 of Liu [9] are the special cases of Theorem 3.1.

3. In case $u_{i}=v_{i}=0, \psi=I, A_{i}=B_{i}$ and $a_{i}=b_{i}$ for $i=1,2,3$, then Theorem 3.1 reduces to Theorem 4.2 of Liu [9], which in turn, generalizes Theorem 2.3 of Bhakta and Mitra [3] and Theorem 4 of Chang [6].

4. If we replace $o p t=\sup , A_{1}=B_{1}=p=q=u_{2}=v_{2}=0$, then Theorem 3.1 reduces to Theorem 3.1 of Liu et al. [15].

5. Theorem 3.2 and Theorem 3.3 of Liu et al. [15] are the special cases of Theorem 3.1.

The following example demonstrates that Theorem 3.1 generalizes and unify the works of $[3,6,9,12,15]$.

Example 3.1. Let $X=Y=\mathbb{R}, S=D=[1, \infty)$. Define $a_{i}, b_{i}: S \times D \rightarrow S$, $u_{i}, v_{i}: S \times D \rightarrow \mathbb{R}$ and $A_{i}, B_{i}: S \times D \times \mathbb{R} \rightarrow \mathbb{R}$ for $i=1,2,3$, and $\varphi, \psi:$ $R^{+} \rightarrow R^{+}$by 


$$
\begin{aligned}
& \psi(t)=2 t^{2}, \varphi(t)=\frac{t}{2}, p(x, y)=\frac{x^{2}}{1+x+3 y}, q(x, y)=\frac{x^{2}}{1+x y} \\
& u_{1}(x, y)=\frac{x^{2}}{1+x+y^{2}}, u_{2}(x, y)=\frac{x^{2} \sin (x+y)}{1+y^{2}}, u_{3}(x, y)=\frac{x^{2} \cos \left(2 x^{3} y\right)}{1+y}, \\
& v_{1}(x, y)=\left\{\begin{array}{ll}
x^{2}|\sin (3 y+1)|, & \text { if } x<2 \\
\frac{x^{2}}{1+y^{2}} & \text { if } x \geq 2,
\end{array} \quad a_{1}(x, y)= \begin{cases}\frac{x}{2} \sin \left(3 x^{2} y\right), & \text { if } x<3 \\
\frac{x+y}{2+x+y} & \text { if } x \geq 3,\end{cases} \right. \\
& v_{2}(x, y)=x^{2}\left|\sin \left(\frac{x^{2}+1}{1+y^{2}}\right)\right|, v_{3}(x, y)=\frac{x^{2}}{1+|\sin (2 x+7 y+1)|}, \\
& a_{2}(x, y)=\frac{x \cos ^{2}(2 x+y)}{3+y}, a_{3}(x, y)=\frac{x}{2+y^{2}}, b_{1}(x, y)=\frac{x \cos \left(x^{3} y\right)}{2}, \\
& b_{2}(x, y)=\frac{x}{7} \sin \left(\frac{3 x+1}{2 y+1}\right), b_{3}(x, y)=\frac{x \sin ^{2}\left(3 x y^{2}+1\right)}{3} \text {, } \\
& B_{1}(x, y, z)=\left\{\begin{array}{ll}
0, & \text { if } z \leq 0 \\
\frac{z}{1+x+y^{2}} & \text { if } z>0,
\end{array} \quad A_{1}(x, y, z)= \begin{cases}\frac{z}{2+\left|\sin \left(3 x+y^{2}\right)\right|}, & \text { if } z \leq 0 \\
\frac{z}{x+y^{2}+3} & \text { if } z>0\end{cases} \right. \\
& B_{2}(x, y, z)=\left\{\begin{array}{ll}
0, & \text { if } z \leq 0 \\
\frac{z}{1+x^{2} y+z} & \text { if } z>0,
\end{array} \quad B_{3}(x, y, z)= \begin{cases}0, & \text { if } z \leq 0 \\
\frac{z|\cos (x+2 y)|}{1+x^{2} y} & \text { if } z>0,\end{cases} \right. \\
& A_{2}(x, y, z)=\left\{\begin{array}{ll}
\frac{z}{1+x+y}, & \text { if } z \leq 0 \\
\frac{z}{3+\left|\cos \left(x^{2} y\right)\right|} & \text { if } z>0,
\end{array} \quad A_{3}(x, y, z)= \begin{cases}\frac{z}{x+y^{2}+3}, & \text { if } z \leq 0 \\
\frac{z\left|\sin \left(3 x^{3}+y\right)\right|}{1+x+y^{3}} & \text { if } z>0 .\end{cases} \right.
\end{aligned}
$$

It is easy to verify that all assumptions of Theorem 3.1 are satisfied. Thus it follows from Theorem 3.1 that the system of functional equations (3) possesses coincidence solutions in $B B(S)$.

Clearly, the results in [3, 6, 9, 12, 15] are not applicable for the system of functional equations (3), because

$\left|u_{1}(x, y)\right|=\frac{x^{2}}{1+x+y^{2}}>0,|p(x, y)|=\frac{x^{2}}{1+x+3 y}>0$, for $\operatorname{all}(x, y) \in S \times D$

and

$$
\max \{|p(x, y)|,|q(x, y)|\} \leq\|x\| \text { does not holds for }(x, y)=(4,1) \text {. }
$$




\section{Existence and uniqueness of solutions in $\mathrm{BC}(\mathrm{S})$ and $\mathrm{B}(\mathrm{S})$}

Now we shall discuss existence and uniqueness of solutions in $B C(S)$ and $B(S)$.

Theorem 4.1. Let $u, p_{i}: S \times D \rightarrow \mathbb{R}, a_{i}: S \times D \rightarrow S$ and $A_{i}: S \times D \times \mathbb{R} \rightarrow \mathbb{R}$ for $i=1,2,3$ be mappings and let $\varphi \in \Phi_{3}$ and $\psi \in \Phi_{4}$ be such that

$\left(C_{1}\right) u, v, p_{i}$ and $A_{i}$ are bounded for $i=1,2,3$.

$\left(C_{2}\right)$ for each $x_{0} \in S, u(x, y) \rightarrow u\left(x_{0}, y\right), v(x, y) \rightarrow v\left(x_{0}, y\right), p_{i}(x, y) \rightarrow$ $p_{i}\left(x_{0}, y\right), a_{i}(x, y) \rightarrow a_{i}\left(x_{0}, y\right)$, as $x \rightarrow x_{0}$ uniformly for $y \in D$ for $i=1,2,3$.

$\left(C_{3}\right) \max \left\{\left|A_{i}(x, y, z)-A_{i}\left(x_{0}, y, z\right)\right|: i=1,2,3\right\} \leq \varphi\left(\left\|x-x_{0}\right\|\right)$ for $x, x_{0} \in$ $S, y \in D, z \in \mathbb{R}$.

$\left(C_{4}\right) \max \left\{\left|A_{i}(x, y, z)-A_{i}\left(x, y, z_{0}\right)\right|: i=1,2,3\right\} \leq \psi\left(\left|z-z_{0}\right|\right)$ for $x \in S, y \in$ $D, z, z_{0} \in \mathbb{R}$.

Then the functional equation (1) possesses a unique solution $w \in B C(S)$ and $\left\{H^{n} h\right\}_{n \geq 1}$ converges to $w$ for each $h \in B C(S)$, where $H$ is defined by

$H h(x)=\operatorname{opt}_{y \in D}\left\{u(x, y)+o p t\left\{v(x, y), p_{i}(x, y)+A_{i}\left(x, y, h\left(a_{i}(x, y)\right)\right): i=1,2,3\right\}\right\}$,

$$
x \in S \text {. }
$$

Proof. Let $x_{0} \in S$ and $h \in B C(S)$. It is clear that $\left(C_{1}\right)$ implies that $H h$ is bounded. In view of $\left(C_{2}\right), \varphi \in \Phi_{3}$ and $\psi \in \Phi_{4}$, we know that for a given $\epsilon>0$, there exist $\delta_{1}>0, \delta_{2}>0$ and $\delta_{3}>0$ so that

$$
\begin{aligned}
& \varphi\left(\left\|x-x_{0}\right\|\right)<\frac{\epsilon}{5} \text { for } x \in S \text { with }\left\|x-x_{0}\right\|<\delta_{1}, \\
& \psi\left(\delta_{1}\right)<\frac{\epsilon}{5}, \\
& \left|u(x, y)-u\left(x_{0}, y\right)\right|<\frac{\epsilon}{5} \text { for }(x, y) \in S \times D \text { with }\left\|x-x_{0}\right\|<\delta_{1}, \\
& \left|v(x, y)-v\left(x_{0}, y\right)\right|<\frac{\epsilon}{5} \text { for }(x, y) \in S \times D \text { with }\left\|x-x_{0}\right\|<\delta_{1}, \\
& \max \left\{\left|p_{i}(x, y)-p_{i}\left(x_{0}, y\right)\right|: i=1,2,3\right\}<\frac{\epsilon}{5} \text { for }(x, y) \in S \times D \text { with }\left\|x-x_{0}\right\|<\delta_{1}, \\
& \left|h(x)-h\left(x_{0}\right)\right|<\delta_{1} \text { for } x \in S \text { with }\left\|x-x_{0}\right\|<\delta_{2}, \\
& \max \left\{\left|a_{i}(x, y)-a_{i}\left(x_{0}, y\right)\right|: i=1,2,3\right\}<\delta_{2} \text { for }(x, y) \in S \times D \text { with }\left\|x-x_{0}\right\|<\delta_{3} .
\end{aligned}
$$


On an account of (41), (45) and (46), we conclude that

$$
\begin{aligned}
& \psi\left(\sup _{y \in D}\left\{\max \left\{\left|h\left(a_{i}(x, y)\right)-h\left(a_{i}\left(x_{0}, y\right)\right)\right|: i=1,2,3\right\}\right\}\right) \\
& \leq \psi\left(\delta_{1}\right)<\frac{\epsilon}{5}, \text { with }\left\|x-x_{0}\right\|<\delta_{3} .
\end{aligned}
$$

Set $\delta=\min \left\{\delta_{1}, \delta_{3}\right\}$. In view of $\left(C_{3}\right),\left(C_{4}\right),(40)-(47)$, we deduce that for $x \in S$ with $\left\|x-x_{0}\right\|<\delta$,

$$
\begin{aligned}
& \left|H h(x)-H h\left(x_{0}\right)\right| \\
& =\operatorname{opt}_{y \in D}\left\{u(x, y)+o p t\left\{v(x, y), p_{i}(x, y)+A_{i}\left(x, y, h\left(a_{i}(x, y)\right)\right): i=1,2,3\right\}\right\} \\
& -o p t_{y \in D}\left\{u\left(x_{0}, y\right)+o p t\left\{v\left(x_{0}, y\right), p_{i}\left(x_{0}, y\right)+A_{i}\left(x_{0}, y, h\left(a_{i}\left(x_{0}, y\right)\right)\right): i=1,2,3\right\}\right\} \\
& \leq \sup _{y \in D}\left\{\left|u(x, y)-u\left(x_{0}, y\right)\right|+\max \left\{\left|v(x, y)-v\left(x_{0}, y\right)\right|,\left|p_{i}(x, y)-p_{i}\left(x_{0}, y\right)\right|\right.\right. \\
& \left.\left.+\left|A_{i}(x, y, h(a(x, y)))-A_{i}\left(x_{0}, y, h\left(a\left(x_{0}, y\right)\right)\right)\right|: i=1,2,3\right\}\right\} \\
& \leq \sup _{y \in D}\left\{\left|u(x, y)-u\left(x_{0}, y\right)\right|+\max \left\{\left|v(x, y)-v\left(x_{0}, y\right)\right|,\left\{\left|p_{i}(x, y)-p_{i}\left(x_{0}, y\right)\right|\right.\right.\right. \\
& : i=1,2,3\}+\left\{\left|A_{i}(x, y, h(a(x, y)))-A_{i}\left(x_{0}, y, h(a(x, y))\right)\right|\right. \\
& \left.\left.\left.+\left|A_{i}\left(x_{0}, y, h(a(x, y))\right)-A_{i}\left(x_{0}, y, h\left(a\left(x_{0}, y\right)\right)\right)\right|: i=1,2,3\right\}\right\}\right\} \\
& <\epsilon \text {, }
\end{aligned}
$$

which implies that $H h$ is continuous at $x_{0}$. Thus $H$ is a self mapping on $B C(S)$. Given $\epsilon>0, x \in S$ and $h, g \in B C(S)$. Suppose that opt $_{y \in D}=$ $\sup _{y \in D}$, then there exist $y, z \in D$ such that

$$
\begin{aligned}
& H h(x)<u(x, y)+\operatorname{opt}\left\{v(x, y), p_{i}(x, y)+A_{i}\left(x, y, h\left(a_{i}(x, y)\right)\right): i=1,2,3\right\}+\epsilon, \\
& H g(x)<u(x, z)+\operatorname{opt}\left\{v(x, z), p_{i}(x, z)+A_{i}\left(x, z, g\left(a_{i}(x, z)\right)\right): i=1,2,3\right\}+\epsilon, \\
& H h(x) \geq u(x, z)+\operatorname{opt}\left\{v(x, z), p_{i}(x, z)+A_{i}\left(x, z, h\left(a_{i}(x, z)\right)\right): i=1,2,3\right\}, \\
& H g(x) \geq u(x, y)+\operatorname{opt}\left\{v(x, y), p_{i}(x, y)+A_{i}\left(x, y, g\left(a_{i}(x, y)\right)\right): i=1,2,3\right\} .
\end{aligned}
$$

Using (48) and $\left(C_{4}\right)$, we get

$$
\begin{aligned}
|H h(x)-H g(x)|< & \max \left\{\left|A_{i}\left(x, y, h\left(a_{i}(x, y)\right)\right)-A_{i}\left(x, y, g\left(a_{i}(x, y)\right)\right)\right|\right. \\
& \left.\left|A_{i}\left(x, z, h\left(a_{i}(x, z)\right)\right)-A_{i}\left(x, z, g\left(a_{i}(x, z)\right)\right)\right|: i=1,2,3\right\}+\epsilon, \\
\leq & \psi\left(\operatorname { m a x } \left\{\left|h\left(a_{i}(x, y)\right)-g\left(a_{i}(x, y)\right)\right|,\left|h\left(a_{i}(x, z)\right)-g\left(a_{i}(x, z)\right)\right|\right.\right. \\
& : i=1,2,3\})+\epsilon, \\
\leq & \psi\left(\|h-g\|_{1}\right)+\epsilon,
\end{aligned}
$$

which gives

$$
\|H h-H g\|_{1} \leq \psi\left(\|h-g\|_{1}\right)+\epsilon .
$$

Similarly, we conclude that (49) holds for $o p t_{y \in D}=\inf _{y \in D}$. Now as $\epsilon \rightarrow 0^{+}$ in (49), we obtain

$$
\|H h-H g\|_{1} \leq \psi\left(\|h-g\|_{1}\right) .
$$


Thus Lemma 2.1 ensures that $h$ has a unique fixed point $w \in B C(S)$ and $\left\{H^{n} h\right\}_{n \geq 1}$ converges to $w$ for any $h \in B C(S)$. Obviously, $w$ is the unique solution of the functional equation $(1)$ in $B C(S)$. This completes the proof.

It follows from the proof of Theorem 4.1 that the following result also holds.

Theorem 4.2. Let $u, p_{i}: S \times D \rightarrow \mathbb{R}, a_{i}: S \times D \rightarrow S$ and $A_{i}: S \times D \times \mathbb{R} \rightarrow$ $\mathbb{R}$ for $i=1,2,3$ be mappings and let $\psi$ be in $\Phi_{7}$ satisfying $\left(C_{1}\right)$ and $\left(C_{4}\right)$. Then the functional equation (1) possesses a unique solution $w \in B C(S)$ and $\left\{H^{n} h\right\}_{n \geq 1}$ converges to $w$ for each $h \in B C(S)$, where $H$ is defined by (39).

Remark 4.3. 1. In case, $u=0$ and $p_{3}=A_{3}=0$, Theorem 4.2 reduces to Theorem 3.2 of Pathak and Deepmala [17].

2. In case $v=0, p_{3}=A_{3}=0$, Theorem 4.2 reduces to Theorem 4.1 of Liu et al. [12].

3. In case $v=0, p_{1}=p_{2}=p_{3}=0, A_{1}=A_{2}=A_{3}$ and $a_{1}=a_{2}=a_{3}$, Theorem 4.2 reduces to a result which generalizes Theorem 2.1 of Bhakta and Mitra [3].

4. If we replace $v=0, p_{1}=p_{2}=p_{3}=0$ and $A_{1}=A_{2}=A_{3}$, then Theorem 4.1 reduces to Theorem 3.1 of Liu et al. [16].

5. If we replace $o p t_{y \in D}=\sup _{y \in D}$ and $u=p_{1}=p_{2}=A_{1}=0$, then Theorem 4.2 reduces to a result which generalizes Theorem 2.1 of Liu et al. [15].

6. If we put $v=p_{1}=p_{2}=p_{3}=0$ and $A_{1}=A_{2}=A_{3}$, then Theorem 4.2 reduces to Theorem 3.2 of Liu et al. [16] and Theorem 3.1 of Liu and Kang [10].

7. Theorem 4.2 extends and improves the results of Bellman [1].

The example below shows that Theorem 4.2 generalizes and unifies the results of $[1,3,10,12,15,16,17]$.

Example 4.1. Let $X=Y=\mathbb{R}, S=[1, \infty), D=R^{+}=[0, \infty), \varphi(t)=2 t$ and $\psi(t)=\frac{t}{3}$, then Theorem 4.2 ensures that the functional equation

$$
\begin{aligned}
& f(x)=\text { opt }_{y \in D}\left\{1 + \frac { 1 } { x + 2 y ^ { 2 } } + \text { opt } \left\{1+\frac{x}{1+x+y^{2}}, \frac{x^{2}}{x+y^{2}}+\frac{1}{1+x^{2}+y^{2}}\right.\right. \\
& +\frac{1}{3} \sin \left(f\left(2 x^{2} y\right)\right), \frac{(x+y)}{1+3(x+y)}+\frac{1}{x^{2}+y}+\frac{1}{3+3|f(\sin (2 x+3 y))|}, \\
& \left.\left.\frac{x^{3}}{x+y^{4}}+\frac{1}{1+x^{2}+2 y^{2}}+\frac{f(5+\sin (7 x-3 y))}{3+3[f(5+\sin (7 x-3 y))]^{2}}\right\}\right\}, \quad \forall x \in S .
\end{aligned}
$$


possesses a unique solution in $B(S)$.

Since, here we have

$$
\begin{gathered}
u(x, y)=1+\frac{1}{x+2 y^{2}}, v(x, y)=1+\frac{x}{1+x+y^{2}}, p_{1}(x, y)=\frac{x^{2}}{x+y^{2}}, \\
p_{2}(x, y)=\frac{(x+y)}{1+3(x+y)}, p_{3}(x, y)=\frac{x^{3}}{x+y^{4}}, A_{1}(x, y, z)=\frac{1}{1+x^{2}+y^{2}}+\frac{1}{3} \sin z, \\
A_{2}(x, y, z)=\frac{1}{x^{2}+y}+\frac{1}{3+3|z|}, A_{3}(x, y, z)=\frac{1}{1+x^{2}+2 y^{2}}+\frac{z}{3+3 z^{2}}
\end{gathered}
$$

and

$$
\begin{aligned}
& \left|A_{i}(x, y, z)-A_{i}\left(x_{0}, y, z\right)\right| \leq 2\left|x-x_{0}\right|, \quad \text { for } i=1,2,3 \\
& \left|A_{i}(x, y, z)-A_{i}\left(x, y, z_{0}\right)\right| \leq \frac{1}{3}\left|z-z_{0}\right|, \quad \text { for } i=1,2,3
\end{aligned}
$$

all conditions of Theorem 4.2 are satisfied. Hence, $(50)$ has a solution in $B(S)$.

However, the corresponding results in $[1,3,10,12,15,16,17]$ are not applicable for the functional equation (50), because

$|u(x, y)|=1+\frac{1}{x+2 y^{2}}>0,|v(x, y)|=1+\frac{x}{1+x+y^{2}}>0$, for all $(x, y) \in S \times D$

\section{Existence and uniqueness of solutions in $\mathrm{BB}(\mathrm{S})$}

Here we shall discuss properties of solutions in $B B(S)$.

Theorem 5.1. Let $u, v, r, t_{i}, p, q_{i}: S \times D \rightarrow \mathbb{R}$ and $s, c, a_{i}: S \times D \rightarrow S$ for $i=1,2,3$, and satisfy the following conditions

$\left(B_{1}\right) u, v$ and $t_{i}$ are bounded on $\bar{B}(0, k) \times D$ for $k \geq 1$ and $i=1,2,3$,

$\left(B_{2}\right) \max \left\{\|c(x, y)\|,\|s(x, y)\|,\left\|a_{i}(x, y)\right\|: i=1,2,3\right\} \leq\|x\|$ for $(x, y) \in$ $S \times D$

$\left(B_{3}\right)$ there exists a constant $\alpha$ such that $\sup _{(x, y) \in S \times D}\left\{|r(x, y)|+\max \left\{|p(x, y)|,\left|q_{i}(x, y)\right|: i=1,2,3\right\}\right\} \leq \alpha<1$,

then the functional equation (2) possesses a unique solution $w \in B B(S)$ and the sequences $\left\{f_{n}\right\}_{n \geq 0}$ and $\left\{w_{n}\right\}_{n \geq 0}$ generated by Algorithm 1 and Algorithm 2, respectively, converge to $f$ and have the error bounds:

$$
d_{k}\left(f_{n+1}, w\right) \leq e^{-(1-\alpha) \sum_{i=0}^{n} \alpha_{i}} d_{k}\left(f_{0}, w\right), \quad \forall n \geq 0, k \in N,
$$


and

$$
d_{k}\left(w_{n+1}, w\right) \leq \frac{\alpha^{n+1}}{1-\alpha} d_{k}\left(w_{1}, w_{0}\right), \quad \forall n \geq 0, k \in N .
$$

Proof. Define a mapping $H: B B(S) \longrightarrow B B(S)$ by

$$
\begin{aligned}
& H h(x)=\text { opt }_{y \in D}\{u(x, y)+r(x, y) h(c(x, y)) \\
& \left.\quad+\operatorname{opt}\left\{v(x, y), p(x, y) h(s(x, y)), t_{i}(x, y)+q_{i}(x, y) h\left(a_{i}(x, y)\right): i=1,2,3\right\}\right\} .
\end{aligned}
$$

It follows from $\left(B_{1}\right)$ and $\left(B_{2}\right)$ that for each $k \geq 1$ and $h \in B B(S)$, there exists $\beta(k)>0$ and $\eta(k, h)>0$ such that

$$
\begin{gathered}
\sup _{(x, y) \in \bar{B}(0, k) \times D}\left\{|u(x, y)|,|v(x, y)|,\left|t_{i}(x, y)\right|: i=1,2,3\right\} \leq \beta(k) \\
\sup \left\{|h(c(x, y))|,|h(s(x, y))|,\left|h\left(a_{i}(x, y)\right)\right|: i=1,2,3\right\} \leq \eta(k, h) .
\end{gathered}
$$

By virtue of $\left(B_{3}\right),(54)$ and $(55)$, for $x \in \bar{B}(0, k)$, we have

$$
\begin{aligned}
|H h(x)| & \leq \sup _{y \in D}\{|u(x, y)|+|r(x, y)||h(c(x, y))|+\max \{|v(x, y)|,|p(x, y)| \\
& \left.\left.\leq 2 \beta(k(x, y))|,| t_{i}(x, y)|+| q_{i}(x, y)|| h\left(a_{i}(x, y)\right) \mid: i=1,2,3\right\}\right\}, \\
& 2 \beta(k, h) .
\end{aligned}
$$

This means that $H$ is a self mapping on $B B(S)$.

Let $\epsilon>0, x \in \bar{B}(0, k), h, g \in B B(S)$ and $k \geq 1$. Suppose that, opt $_{y \in D}=\sup _{y \in D}$. Then $\exists y, z \in D$ such that

$$
\begin{aligned}
H h(x)< & u(x, y)+r(x, y) h(c(x, y))+o p t\{v(x, y), p(x, y) h(s(x, y)), \\
& \left.t_{i}(x, y)+q_{i}(x, y) h\left(a_{i}(x, y)\right): i=1,2,3\right\}+\epsilon, \\
H g(x)< & u(x, z)+r(x, z) g(c(x, z))+o p t\{v(x, z), p(x, z) g(s(x, z)), \\
& \left.t_{i}(x, z)+q_{i}(x, z) g\left(a_{i}(x, z)\right): i=1,2,3\right\}+\epsilon, \\
H h(x) \geq \quad & u(x, z)+r(x, z) h(c(x, z))+\operatorname{opt}\{v(x, z), p(x, z) h(s(x, z)), \\
& \left.t_{i}(x, z)+q_{i}(x, z) h\left(a_{i}(x, z)\right): i=1,2,3\right\}, \\
H g(x) \geq & u(x, y)+r(x, y) g(c(x, y))+o p t\{v(x, y), p(x, y) g(s(x, y)), \\
& \left.t_{i}(x, y)+q_{i}(x, y) g\left(a_{i}(x, y)\right): i=1,2,3\right\} .
\end{aligned}
$$

Using (56), we find 


$$
\begin{aligned}
& H h(x)-H g(x) \\
& <r(x, y)(h(c(x, y))-g(c(x, y))) \\
& +o p t\left\{v(x, y), p(x, y) h(s(x, y)), t_{i}(x, y)+q_{i}(x, y) h\left(a_{i}(x, y)\right): i=1,2,3\right\} . \\
& \text {-opt }\left\{v(x, y), p(x, y) g(s(x, y)), t_{i}(x, y)+q_{i}(x, y) g\left(a_{i}(x, y)\right): i=1,2,3\right\}+\epsilon \text {, } \\
& \leq|r(x, y)||(h(c(x, y))-g(c(x, y)))|+\max \{|p(x, y)||h(s(x, y))-g(s(x, y))|, \\
& \left.\left|q_{i}(x, y)\right|\left|h\left(a_{i}(x, y)\right)-g\left(a_{i}(x, y)\right)\right|: i=1,2,3\right\}+\epsilon \text {, } \\
& \leq\left[|r(x, y)|+\max \left\{|p(x, y)|,\left|q_{i}(x, y)\right|: i=1,2,3\right\}\right] \max \{|(h(c(x, y))-g(c(x, y)))| \text {, } \\
& \left.|h(s(x, y))-g(s(x, y))|,\left|h\left(a_{i}(x, y)\right)-g\left(a_{i}(x, y)\right)\right|: i=1,2,3\right\}+\epsilon \text {, } \\
& \leq \alpha \max \{|(h(c(x, y))-g(c(x, y)))|,|h(s(x, z))-g(s(x, z))| \text {, } \\
& \left.\left|h\left(a_{i}(x, y)\right)-g\left(a_{i}(x, y)\right)\right|: i=1,2,3\right\}+\epsilon \\
& H h(x)-H g(x) \\
& >\quad r(x, z)(h(c(x, z))-g(c(x, z))) \\
& +\operatorname{opt}\left\{v(x, z), p(x, z) h(s(x, z)), t_{i}(x, z)+q_{i}(x, z) h\left(a_{i}(x, z)\right): i=1,2,3\right\} . \\
& -o p t\left\{v(x, z), p(x, z) g(s(x, z)), t_{i}(x, z)+q_{i}(x, z) g\left(a_{i}(x, z)\right): i=1,2,3\right\}-\epsilon \text {, } \\
& \geq-|r(x, z)| \mid(h(c(x, z))-g(c(x, z)))-\max \{|p(x, z)||h(s(x, z))-g(s(x, z))| \text {, } \\
& \left.\left|q_{i}(x, z)\right|\left|h\left(a_{i}(x, z)\right)-g\left(a_{i}(x, z)\right)\right|: i=1,2,3\right\}-\epsilon, \\
& \geq\left[-|r(x, z)|-\max \left\{|p(x, z)|,\left|q_{i}(x, z)\right|: i=1,2,3\right\}\right] \\
& \max \{|(h(c(x, z))-g(c(x, z)))|,|h(s(x, z))-g(s(x, z))|, \\
& \left.\left|h\left(a_{i}(x, z)\right)-g\left(a_{i}(x, z)\right)\right|: i=1,2,3\right\}-\epsilon \\
& \geq-\alpha \max \{|(h(c(x, z))-g(c(x, z)))|,|h(s(x, z))-g(s(x, z))| \text {, } \\
& \left.\left|h\left(a_{i}(x, z)\right)-g\left(a_{i}(x, z)\right)\right|: i=1,2,3\right\}-\epsilon \text {, }
\end{aligned}
$$

which imply that

$$
|H h(x)-H g(x)| \leq \alpha d_{k}(h, g)+\epsilon, \quad \forall h, g \in B B(S) .
$$

In a similar way, we can show that (57) holds for opt $t_{y \in D}=\inf _{y \in D}$. As $\epsilon \longrightarrow 0^{+}$ in (57), we get

$$
d_{k}(H h, H g) \leq \alpha d_{k}(h, g), \forall h, g \in B B(S) .
$$

Let $w_{0} \in B B(S)$. It follows from Algorithm 2 that

$$
w_{n+1}=H w_{n}(x), \forall n \geq 0, x \in S .
$$

By a similar argument as in [Theorem 3.3, [8]], we can prove that the functional equation (2) possesses a unique solution $w \in B B(S)$ and have error estimates (51) and (52). This completes the proof of the theorem. 
Remark 5.2. 1. In case $u=r=0$ and $p=0$, Theorem 5.1 reduces to Theorem 4.1 of Pathak and Deepmala [17].

2. If we put $t_{1}=t_{2}=t_{3}=q_{2}=q_{3}=0$ in Theorem 5.1, then we get Theorem 4.1 of Liu et al. [16].

3. In case $r=v=p=t_{3}=q_{3}=0,5.1$ reduces to Theorem 4.2 of Liu et al. [12].

4. If $v=p=t_{3}=q_{3}=0$, then 5.1 reduces to Theorem 3.3 of Liu et al. $[13]$.

5. If $u=r=t_{1}=t_{2}=t_{3}=q_{3}=0$, then 5.1 reduces to Theorem 3.3 of Jiang et al. [8].

6. If we replace $o p t_{y \in D}=\inf _{y \in D}$, opt $=\max$ and $u=r=t_{1}=t_{2}=t_{3}=$ $q_{1}=q_{2}=q_{3}=0$, then 5.1 reduces to Theorem 3.4 of Bhakta and Mitra $[3]$.

7. Theorem 5.1 extends and improves the results of Bellman [1].

The following example demonstrates that Theorem 5.1 generalizes substantially the results obtained in $[1,3,8,12,13,16,17]$.

Example 5.1. Let $X=Y=\mathbb{R}$ and $S=D=R^{+}$. Consider the following functional equation:

$$
\begin{aligned}
& f(x)=\text { opt }_{y \in D}\left\{1+\frac{x^{4}}{1+x^{2}+y^{2}}+\frac{\cos \left(x+y^{2}\right)}{3+x y} f\left(\frac{x^{3}}{1+x^{2} y}\right)+\right. \\
& o p t_{y \in D}\left\{1+\frac{1}{3+x^{2}+y^{3}}, \frac{x y^{2}}{4+x y^{2}} f\left(\frac{x^{2} \sin \left(x^{2} y\right)}{1+x y}\right),\left(1+\sin \left(x^{2}+3 y\right)\right)+\right. \\
& \frac{\sin (x+2 y)}{4+x^{2}+y^{3}} f\left(\frac{2 x}{4+x+y}\right), \frac{1}{1+x y^{2}}+\frac{1}{4+x+y^{3}} f\left(\frac{x^{2}}{1+x+y}\right), \\
& \left.\left.\frac{x}{x+y^{2}}+\frac{x^{3} y^{3}}{4+x^{3} y^{3}} f\left(\frac{x^{2} \sin ^{2}(x+2 y)}{1+x+y^{2}}\right)\right\}\right\}, \quad \forall x \in S .
\end{aligned}
$$

Clearly, for all $(x, y) \in S \times D$,

$$
\begin{gathered}
\max \left\{\frac{x^{3}}{1+x^{2} y}, \frac{x^{2}\left|\sin \left(x^{2} y\right)\right|}{1+x y}, \frac{2 x}{4+x+y}, \frac{x^{2}}{1+x+y},\right. \\
\left.\frac{x^{2}\left|\sin ^{2}(x+2 y)\right|}{1+x+y^{2}}\right\} \leq x
\end{gathered}
$$


and

$$
\begin{gathered}
\sup _{(x, y) \in S \times D}\left\{\frac{\left|\cos \left(x+y^{2}\right)\right|}{3+x y}+\max \left\{\frac{x y^{2}}{4+x y^{2}}, \frac{|\sin (x+2 y)|}{4+x^{2}+y^{3}}, \frac{1}{4+x+y^{3}},\right.\right. \\
\left.\left.\frac{x^{3} y^{3}}{4+x^{3} y^{3}},\right\}\right\}<1 .
\end{gathered}
$$

It follows from Theorem 5.1 that the functional equation (59) possesses a unique solution in $B B(S)$. However, the results given in $[1,3,8,12,13,16,17]$ are not applicable for the functional equation (59), because

$$
|u(x, y)|=\left(1+\frac{x^{4}}{1+x^{2}+y^{2}}\right)>0,|v(x, y)|=\left(1+\frac{1}{3+x^{2}+y^{3}}\right)>0
$$

and

$$
\left|t_{1}(x, y)\right|=1+\left|\sin \left(x^{2}+3 y\right)\right|>0, \quad \forall(x, y) \in S \times D .
$$

Theorem 5.3. Let $u, v, r, t_{i}, p, q_{i}: S \times D \rightarrow \mathbb{R}$ and $s, c, a_{i}: S \times D \longrightarrow S$ for $i=1,2,3$, and let $(\varphi, \psi)$ be in $\left(\Phi_{6}\right)$ satisfying the following conditions:

$\left(B_{4}\right) \sup _{y \in D}\left\{|u(x, y)|+\max \left\{|v(x, y)|,\left|t_{i}(x, y)\right|: i=1,2,3\right\}\right\} \leq \psi(\|x\|), \forall x \in$ $S$.

$\left(B_{5}\right) \max \left\{\|c(x, y)\|,\|s(x, y)\|,\left\|a_{i}(x, y)\right\|: i=1,2,3\right\} \leq \varphi(\|x\|), \forall(x, y) \in$ $S \times D$.

$\left(B_{6}\right) \sup _{y \in D}\left\{|r(x, y)|+\max \left\{|p(x, y)|,\left|q_{i}(x, y)\right|: i=1,2,3\right\}\right\} \leq 1, \forall x \in S$.

Then the functional equation (2) possesses a solution $w \in B B(S)$ which satisfies the following properties:

$\left(B_{7}\right)$ The sequence $\left\{w_{n}\right\}_{n \geq 0}$ defined by

$w_{0}(x)=o t_{y \in D}\left\{u(x, y)+\max \left\{v(x, y), t_{i}(x, y): i=1,2,3\right\}\right\}, \forall x \in S$

$w_{n}(x)=$ opt $_{y \in D}\left\{u(x, y)+r(x, y) w_{n-1}(c(x, y))\right.$

$+\operatorname{opt}\left\{v(x, y), p(x, y) w_{n-1}(s(x, y))\right.$,

$\left.\left.t_{i}(x, y)+q_{i}(x, y) w_{n-1}\left(a_{i}(x, y)\right): i=1,2,3\right\}\right\}$,

$\forall x \in S, n \geq 1$ converges to $w$.

$\left(B_{8}\right) \lim _{n \rightarrow \infty} w\left(x_{n}\right)=0$ for any $x_{0} \in S$,

$\left\{y_{n}\right\}_{n \geq 1} \subset D$ and $x_{n} \in\left\{c\left(x_{n-1}, y_{n}\right), s\left(x_{n-1}, y_{n}\right), a_{i}\left(x_{n-1}, y_{n}\right): i=\right.$ $1,2,3\}, \forall n \in N$.

$\left(B_{9}\right) w$ is unique with respect to condition $\left(B_{8}\right)$. 
Proof. Since $(\varphi, \psi)$ is in $\left(\Phi_{6}\right)$, it is easy to verify that

$$
\varphi(t)<t \quad \text { for } \quad t<0 \text {. }
$$

We assert that the mapping $H$ defined in (53) is non-expansive on $B B(S)$. For this, in view of $(60)$ and $\left(B_{5}\right)$, we have

$\max \left\{\|c(x, y)\|,\|s(x, y)\|,\left\|a_{i}(x, y)\right\|: i=1,2,3\right\} \leq \varphi(\|x\|)<k$, for $(x, y) \in$ $\bar{B}(0, k) \times D$,

which implies that $\exists$ a constant $\theta(k, h)>0$ such that

$$
\begin{gathered}
\max \left\{|h(c(x, y))|,|h(s(x, y))|,\left|h\left(a_{i}(x, y)\right)\right|: i=1,2,3\right\} \\
\leq \theta(k, h), \text { for }(x, y) \in \bar{B}(0, k) \times D .
\end{gathered}
$$

By virtue of $\left(B_{4}\right),\left(B_{6}\right)$, (53), Lemma 2.2 and Lemma 2.3 , we deduce that $|H h(x)| \leq \sup _{y \in D}\{|u(x, y)|+|r(x, y)||h(c(x, y))|+\max \mid\{|v(x, y)|$, $\left.\left.|p(x, y)||h(s(x, y))|,\left|t_{i}(x, y)\right|+\left|q_{i}(x, y)\right|\left|h\left(a_{i}(x, y)\right)\right|: i=1,2,3\right\}\right\}$ $\leq \sup _{y \in D}\left\{|u(x, y)|+\max \left\{|v(x, y)|,\left|t_{i}(x, y)\right|: i=1,2,3\right\}+\{|r(x, y)|\right.$ $\left.+\max \left\{|p(x, y)|,\left|q_{i}(x, y)\right|: i=1,2,3\right\}\right\}$ $\left.\max \left\{|h(c(x, y))|,|h(s(x, y))|,\left|h\left(a_{i}(x, y)\right)\right|: i=1,2,3\right\}\right\}$

$$
\leq \psi(k)+\theta(k, h) \text { for } x \in \bar{B}(0, k) \text {. }
$$

Thus $H$ is a self mapping on $B B(S)$.

Now as in the proof of Theorem 5.1, we can immediately conclude that $h, g \in$ $B B(S)$ and for all $k \geq 1$,

$$
d_{k}(H h, H g) \leq d_{k}(h, g)
$$

which yields that

$$
d(H h, H g)=\sum_{k=1}^{\infty} \frac{1}{2^{k}} \frac{d_{k}(H h, H g)}{1+d_{k}(H h, H g)} \leq \sum_{k=1}^{\infty} \frac{1}{2^{k}} \frac{d_{k}(h, g)}{1+d_{k}(h, g)}=d(h, g)
$$

for $h, g \in B B(S)$. Thus $H$ is nonexpansive.

Now we assert that for each $n \geq 0$,

$$
\left|w_{n}(x)\right| \leq \sum_{j=0}^{n} \psi\left(\varphi^{j}(\|x\|)\right), x \in S
$$

For this, by $\left(B_{4}\right)$ we have

$$
\begin{aligned}
\left|w_{0}(x)\right| & \leq \sup _{y \in D}\left\{|u(x, y)|+\max \left\{|v(x, y)|,\left|t_{i}(x, y)\right|: i=1,2,3\right\}\right\} \\
& \leq \psi(\|x\|), x \in S
\end{aligned}
$$

i.e., (62) is true for $n=0$. Suppose that (62) holds for some $n \geq 0$, then 
from $\left(B_{4}\right)-\left(B_{6}\right)$ we find

$$
\begin{aligned}
\left|w_{n+1}(x)\right|= & \mid \operatorname{opt}_{y \in D}\left\{u(x, y)+r(x, y) w_{n}(c(x, y))+\operatorname{opt}\{v(x, y), p(x, y)\right. \\
& \left.\left.w_{n}(s(x, y)), t_{i}(x, y)+q_{i}(x, y) w_{n}\left(a_{i}(x, y)\right): i=1,2,3\right\}\right\} \mid \\
\leq & \sup _{y \in D}\left\{|u(x, y)|+|r(x, y)|\left|w_{n}(c(x, y))\right|+\max \{|v(x, y)|,|p(x, y)|\right. \\
& \left.\left.\left|w_{n}(s(x, y))\right|,\left|t_{i}(x, y)\right|+\left|q_{i}(x, y)\right|\left|w_{n}\left(a_{i}(x, y)\right)\right|: i=1,2,3\right\}\right\} \\
\leq & \sup _{y \in D}\left\{|u(x, y)|+\max \left\{|v(x, y)|,\left|t_{i}(x, y)\right|: i=1,2,3\right\}\right. \\
& +\left[|r(x, y)|+\max \left\{|p(x, y)|,\left|q_{i}(x, y)\right|: i=1,2,3\right\}\right] \\
& \left.\max \left\{\left|w_{n}(c(x, y))\right|,\left|w_{n}(s(x, y))\right|,\left|w_{n}\left(a_{i}(x, y)\right)\right|: i=1,2,3\right\}\right\} \\
\leq & \psi(\|x\|)+\sum_{j=0}^{n} \psi\left(\varphi^{j+1}(\|x\|)\right) \\
\leq & \sum_{j=0}^{n+1} \psi\left(\varphi^{j}(\|x\|)\right),
\end{aligned}
$$

Hence (62) holds for all $n \geq 0$.

Next we claim that $\left\{w_{n}\right\}_{n \geq 0}$ is a cauchy sequence in $B B(S)$. Given $k \geq 1$ and $x_{0} \in \bar{B}(0, k)$, let $\epsilon>0, n, m \in N$. Suppose that $o p t_{y \in D}=\sup _{y \in D}$. Then we select $y, z \in D$ such that

$$
\begin{aligned}
& w_{n}\left(x_{0}\right)<u\left(x_{0}, y\right)+r\left(x_{0}, y\right) w_{n-1}\left(c\left(x_{0}, y\right)\right)+o p t\left\{v\left(x_{0}, y\right), p\left(x_{0}, y\right) w_{n-1}\right. \\
& \left.\left(s\left(x_{0}, y\right)\right), t_{i}\left(x_{0}, y\right)+q_{i}\left(x_{0}, y\right) w_{n-1}\left(a_{i}\left(x_{0}, y\right)\right): i=1,2,3\right\}+\frac{\epsilon}{2} \\
& w_{n+m}\left(x_{0}\right)<u\left(x_{0}, z\right)+r\left(x_{0}, z\right) w_{n+m-1}\left(c\left(x_{0}, z\right)\right)+o p t\left\{v\left(x_{0}, z\right), p\left(x_{0}, z\right)\right. \\
& w_{n+m-1}\left(s\left(x_{0}, z\right)\right), t_{i}\left(x_{0}, z\right)+q_{i}\left(x_{0}, z\right) w_{n+m-1}\left(a_{i}\left(x_{0}, z\right)\right) \\
& : i=1,2,3\}+\frac{\epsilon}{2} \\
& w_{n}\left(x_{0}\right) \geq u\left(x_{0}, z\right)+r\left(x_{0}, z\right) w_{n-1}\left(c\left(x_{0}, z\right)\right)+\operatorname{opt}\left\{v\left(x_{0}, z\right), p\left(x_{0}, z\right)\right. \\
& \left.w_{n-1}\left(s\left(x_{0}, z\right)\right), t_{i}\left(x_{0}, z\right)+q_{i}\left(x_{0}, z\right) w_{n-1}\left(a_{i}\left(x_{0}, z\right)\right): i=1,2,3\right\} \text {, } \\
& w_{n+m}\left(x_{0}\right) \geq u\left(x_{0}, y\right)+r\left(x_{0}, y\right) w_{n+m-1}\left(c\left(x_{0}, y\right)\right)+o p t\left\{v\left(x_{0}, y\right), p\left(x_{0}, y\right)\right. \\
& \left.w_{n+m-1}\left(s\left(x_{0}, y\right)\right), t_{i}\left(x_{0}, y\right)+q_{i}\left(x_{0}, y\right) w_{n+m-1}\left(a_{i}\left(x_{0}, y\right)\right): i=1,2,3\right\} \text {. }
\end{aligned}
$$

Now in view of $(63),\left(B_{6}\right)$ and Lemma 2.2 , it immediately follows that

$$
\left|w_{n+m}\left(x_{0}\right)-w_{n}\left(x_{0}\right)\right| \leq\left|w_{n+m-1}\left(x_{1}\right)-w_{n-1}\left(x_{1}\right)\right|+\epsilon / 2,
$$

for some $y_{1} \in\{y, z\}$ and $x_{1} \in\left\{c\left(x_{0}, y_{1}\right), s\left(x_{0}, y_{1}\right), a_{i}\left(x_{0}, y_{1}\right): i=1,2,3\right\}$. Similarly, we conclude that the inequality (64) holds for 
opt $_{y \in D}=\inf _{y \in D}$. Proceeding in this way, we select $y_{j} \in D$ and $x_{j} \in$ $\left\{c\left(x_{j-1}, y_{j}\right), s\left(x_{j-1}, y_{j}\right), a_{i}\left(x_{j-1}, y_{j}\right): i=1,2,3\right\}$ for $j=2,3, \ldots, n$ such that

$$
\begin{aligned}
\left|w_{n+m-1}\left(x_{1}\right)-w_{n-1}\left(x_{1}\right)\right| & <\left|w_{n+m-2}\left(x_{2}\right)-w_{n-2}\left(x_{2}\right)\right|+2^{-2} \epsilon \\
\left|w_{n+m-2}\left(x_{2}\right)-w_{n-2}\left(x_{2}\right)\right| & <\left|w_{n+m-3}\left(x_{3}\right)-w_{n-3}\left(x_{3}\right)\right|+2^{-3} \epsilon \\
\ldots & \ldots \ldots \\
\left|w_{m+1}\left(x_{n-1}\right)-w_{1}\left(x_{n-1}\right)\right| & <\left|w_{m}\left(x_{n}\right)-w_{0}\left(x_{n}\right)\right|+2^{-n} \epsilon .
\end{aligned}
$$

It follows from $\left(B_{5}\right),(60),(62),(64)$ and $(65)$ that

$$
\begin{aligned}
\left|w_{n+m}\left(x_{0}\right)-w_{n}\left(x_{0}\right)\right| & <\left|w_{m}\left(x_{n}\right)-w_{0}\left(x_{n}\right)\right|+\sum_{i=1}^{n} 2^{-i} \epsilon \\
& <\left|w_{m}\left(x_{n}\right)\right|+\left|w_{0}\left(x_{n}\right)\right|+\epsilon \\
& \leq \sum_{i=0}^{m} \psi\left(\varphi^{i}\left(\left\|x_{n}\right\|\right)\right)+\psi\left(\left\|x_{n}\right\|\right)+\epsilon \\
& \leq \sum_{i=0}^{m} \psi\left(\varphi^{i+n}\left(\left\|x_{0}\right\|\right)\right)+\psi\left(\varphi^{n}\left(\left\|x_{0}\right\|\right)\right)+\epsilon \\
& \leq \sum_{j=n-1}^{\infty} \psi\left(\varphi^{j}(k)\right)+\epsilon,
\end{aligned}
$$

which implies that

$$
d_{k}\left(w_{n+m}, w_{n}\right) \leq \sum_{j=n-1}^{\infty} \psi\left(\varphi^{j}(k)\right)+\epsilon .
$$

Letting $\epsilon \rightarrow 0^{+}$in the above inequality, we obtain

$d_{k}\left(w_{n+m}, w_{n}\right) \leq \sum_{j=n-1}^{\infty} \psi\left(\varphi^{j}(k)\right)$, which confirms that $\left\{w_{n}\right\}_{n \geq 0}$ is a cauchy sequence in $(B B(S), d)$ since $\sum_{i=0}^{\infty} \psi\left(\varphi^{n}(t)\right)<\infty$, for each $t>0$. Next, suppose $\left\{w_{n}\right\}_{n \geq 0}$ converges to some $w \in B B(S)$. Since $H$ is nonexpansive, it follows that

$$
\begin{aligned}
d(w, H w) & \leq d\left(w, H w_{n}\right)+d\left(H w_{n}, H w\right) \\
\leq & d\left(w, w_{n+1}\right)+d\left(w_{n}, w\right) \\
& \rightarrow 0 \text { as } n \rightarrow \infty
\end{aligned}
$$

i.e., $H w=w$. Thus the functional equation (2) possesses a solution $w$.

Now we shall show that $\left(B_{8}\right)$ holds.

Let $\epsilon>0, x_{0} \in S,\left\{y_{n}\right\}_{n \geq 1} \subset D$ and $x_{n} \in\left\{c\left(x_{n-1}, y_{n}\right), s\left(x_{n-1}, y_{n}\right), a_{i}\left(x_{n-1}, y_{n}\right)\right.$ : $i=1,2,3\}$ for $n \geq 1$. Put $k=\left[\left\|x_{0}\right\|\right]+1$. Then there exists a positive integer $m$ such that

$$
d_{k}\left(w, w_{n}\right)+\sum_{j=n}^{\infty} \psi\left(\varphi^{j}(k)\right)<\epsilon, \text { for } n>m .
$$

By $(62),\left(B_{5}\right)$ and (67), we infer that for $n>m$,

$$
\begin{aligned}
\left|w\left(x_{n}\right)\right| & \leq\left|w\left(x_{n}\right)-w_{n}\left(x_{n}\right)\right|+\left|w_{n}\left(x_{n}\right)\right| \\
& \leq d_{k}\left(w, w_{n}\right)+\sum_{j=0}^{\infty} \psi\left(\varphi^{j}\left(\left\|x_{n}\right\|\right)\right) \\
& \leq d_{k}\left(w, w_{n}\right)+\sum_{j=n}^{\infty} \psi\left(\varphi^{j}(k)\right) \\
& \leq \epsilon,
\end{aligned}
$$

which means that $\lim _{n \rightarrow \infty} w\left(x_{n}\right)=0$. 
Finally, we shall show that $\left(B_{9}\right)$ holds. Suppose that the functional equation (2) possesses another solution $h \in B B(S)$, which satisfies condition $\left(B_{8}\right)$. Let $\epsilon>0$ and $x_{0} \in S$. If $o p t_{y \in D}=\sup _{y \in D}$, then there exists $y, z \in S$ such that

$$
\begin{aligned}
w\left(x_{0}\right)< & u\left(x_{0}, y\right)+r\left(x_{0}, y\right) w\left(c\left(x_{0}, y\right)\right)+o p t\left\{v\left(x_{0}, y\right), p\left(x_{0}, y\right) w\left(s\left(x_{0}, y\right)\right),\right. \\
& \left.t_{i}\left(x_{0}, y\right)+q_{i}\left(x_{0}, y\right) w\left(a_{i}\left(x_{0}, y\right)\right): i=1,2,3\right\}+\frac{\epsilon}{2}, \\
h\left(x_{0}\right)<\quad & u\left(x_{0}, z\right)+r\left(x_{0}, z\right) h\left(c\left(x_{0}, z\right)\right)+o p t\left\{v\left(x_{0}, z\right), p\left(x_{0}, z\right) h\left(s\left(x_{0}, z\right)\right),\right. \\
& \left.t_{i}\left(x_{0}, z\right)+q_{i}\left(x_{0}, z\right) h\left(a_{i}\left(x_{0}, z\right)\right): i=1,2,3\right\}+\frac{\epsilon}{2}, \\
w\left(x_{0}\right) \geq & u\left(x_{0}, z\right)+r\left(x_{0}, z\right) w\left(c\left(x_{0}, z\right)\right)+o p t\left\{v\left(x_{0}, z\right), p\left(x_{0}, z\right) w\left(s\left(x_{0}, z\right)\right),\right. \\
& \left.t_{i}\left(x_{0}, z\right)+q_{i}\left(x_{0}, z\right) w\left(a_{i}\left(x_{0}, z\right)\right): i=1,2,3\right\}, \\
h\left(x_{0}\right) \geq & u\left(x_{0}, y\right)+r\left(x_{0}, y\right) h\left(c\left(x_{0}, y\right)\right)+o p t\left\{v\left(x_{0}, y\right), p\left(x_{0}, y\right) h\left(s\left(x_{0}, y\right)\right),\right. \\
& \left.t_{i}\left(x_{0}, y\right)+q_{i}\left(x_{0}, y\right) h\left(a_{i}\left(x_{0}, y\right)\right): i=1,2,3\right\} .
\end{aligned}
$$

Using Lemma (2.2), $\left(B_{6}\right)$ and (68), we find

$$
\left|w\left(x_{0}\right)-h\left(x_{0}\right)\right| \leq\left|w\left(x_{1}\right)-h\left(x_{1}\right)\right|+\frac{\epsilon}{2} .
$$

for some $y_{1} \in\{y, z\}$ and $x_{1} \in\left\{c\left(x_{0}, y_{1}\right), s\left(x_{0}, y_{1}\right), a_{i}\left(x_{0}, y_{1}\right): i=1,2,3\right\}$. Similarly, we conclude that (69) holds for $o p t_{y \in D}=\inf _{y \in D}$. Proceeding in this way, we select $y_{j} \in D$ and $x_{j} \in\left\{c\left(x_{j-1}, y_{j}\right), s\left(x_{j-1}, y_{j}\right), a_{i}\left(x_{j-1}, y_{j}\right): i=\right.$ $1,2,3\}$ for $j=2,3, \ldots, n$ satisfying

$$
\begin{aligned}
\left|w\left(x_{1}\right)-h\left(x_{1}\right)\right| & <\left|w\left(x_{2}\right)-h\left(x_{2}\right)\right|+2^{-2} \epsilon \\
\left|w\left(x_{2}\right)-h\left(x_{2}\right)\right| & <\left|w\left(x_{3}\right)-h\left(x_{3}\right)\right|+2^{-3} \epsilon \\
\ldots & \ldots \\
\left|w\left(x_{n-1}\right)-h\left(x_{n-1}\right)\right| & <\left|w\left(x_{n}\right)-h\left(x_{n}\right)\right|+2^{-n} \epsilon .
\end{aligned}
$$

Combining (69) and (70), we obtain

$$
\left|w\left(x_{0}\right)-h\left(x_{0}\right)\right|<\left|w\left(x_{n}\right)-h\left(x_{n}\right)\right|+\sum_{j=1}^{n} 2^{-j} \epsilon<\left|w\left(x_{n}\right)-h\left(x_{n}\right)\right|+\epsilon .
$$

Letting $n \rightarrow \infty$ in the above inequalities, by $\left(B_{8}\right)$ we get

$$
\left|w\left(x_{0}\right)-h\left(x_{0}\right)\right| \leq \epsilon .
$$

As $\epsilon \rightarrow 0^{+}$in the above inequality, it follows that $w\left(x_{0}\right)=h\left(x_{0}\right)$. This completes the proof.

Remark 5.4. 1. In case $u=r=0$ and $p=0$, Theorem 5.3 reduces to Theorem 4.3 of Pathak and Deepmala [17]. 
2. In case $t_{1}=t_{2}=t_{3}=q_{2}=q_{3}=0$, Theorem 5.3 reduces to Theorem 4.2 of Liu et al. [16].

3. If $r=v=p=t_{3}=q_{3}=0$, then Theorem 5.3 reduces to Theorem 4.3 of Liu et al. [12].

4. If we replace $o p t_{y \in D}=\sup _{y \in D}, p=q_{1}=1, u=r=q_{2}=q_{3}=t_{2}=t_{3}=$ 0, then Theorem 5.3 reduces to Theorem 2.2 of Liu et al. [15].

5. If $v=p=t_{3}=q_{3}=0$, then Theorem 5.3 reduces to Theorem 3.4 of Liu et al. [13].

6. In case $u=r=q_{3}=t_{1}=t_{2}=t_{3}=0$ and $(\varphi, \psi) \in \Phi_{8}$, Theorem 5.3 reduces to Theorem 3.4 of Jiang et al. [8].

7. If we replace $o p t_{y \in D}=\inf _{y \in D}, p=q_{1}=1$ and $u=r=t_{2}=t_{3}=q_{2}=$ $q_{3}=0$, then Theorem 5.3 reduces to Theorem 3.4 of Liu and Kang [10]

8. Theorem 5.3 extends and improve the results in Bellman [1].

9. If we replace $u(x, y)=\lambda u_{1}(x, y), v(x, y)=(1-\lambda) v_{1}(x, y), r=q_{1}=q_{2}=$ $q_{3}=(1-\lambda), p=0, a_{1}=a_{2}=a_{3}=a, \psi(t)=M t$ and $\varphi \in \Phi_{9}$, for $(x, y) \in S \times D, t \in R^{+}$, where $\lambda \in[0,1]$ and $M$ is a positive constant, then Theorem 5.3 reduces to Theorem 3.1 of Liu and Ume [11], which in turn, generalizes the results in [4] and [3].

The example below demonstrates that Theorem 5.3 generalizes and unifies the results in $[1,3,4,8,10,11,12,13,15,16,17]$.

Example 5.2. Let $X=Y=R, S=D=[1, \infty), \psi(t)=2 t^{3}, \varphi(t)=\frac{t}{2}, \forall t \in$ $R^{+}$. Consider the following functional equation:

$$
\begin{aligned}
& f(x)=\text { opt }_{y \in D}\left\{\frac{x^{4}}{1+x+y}+\frac{1}{3+x+y^{2}} f\left(\frac{x \sin (2 x y)}{2+y}\right)\right. \\
& + \text { opt }_{y \in D}\left\{\frac{x^{3}}{1+\left|\sin \left(3 x y^{2}+4\right)\right|}, \frac{1}{5} \cos \left(x+3 y^{2}\right) f\left(\frac{x^{2}}{2+x+y}\right),\right. \\
& \frac{x^{4}}{1+x y}+\frac{1}{4+x y} f\left(\frac{x \sin (2 x+3 y)}{2+y^{2}}\right), \frac{x^{4}}{1+x+y^{2}}+\frac{1}{7+\left|\sin \left(3 x+y^{2}\right)\right|} \\
& \left.\left.f\left(\frac{x}{2+y}\right), \frac{x^{4}}{1+x+\cos \left(x+2 y^{2}\right)}+\frac{1}{6} \cos (3 x+y+4) f\left(\frac{x}{2+\sin (3 x+y)}\right)\right\}\right\} .
\end{aligned}
$$


Note that

$$
\begin{array}{rlrl}
|u(x, y)| & =\frac{x^{4}}{1+x+y} & & \leq x^{3} \leq \psi(|x|), \\
|v(x, y)| & =\frac{x^{3}}{1+\left|\sin \left(3 x y^{2}+4\right)\right|} & & \leq x^{3} \leq \psi(|x|), \\
\left|t_{1}(x, y)\right| & =\frac{x^{4}}{1+x y} & & \leq x^{3} \leq \psi(|x|), \\
\left|t_{2}(x, y)\right| & =\frac{x^{4}}{1+x+y^{2}} & \leq x^{3} \leq \psi(|x|), \\
\left|t_{3}(x, y)\right| & =\frac{x^{4}}{1+x+\left|\cos \left(x+2 y^{2}\right)\right|} & \leq x^{3} \leq \psi(|x|),
\end{array}
$$

and hence

$\sup _{y \in D}\left\{|u(x, y)|+\max \left\{|v(x, y)|,\left|t_{i}(x, y)\right|: i=1,2,3\right\}\right\} \leq 2 x^{3}=\psi(|x|), \quad \forall x \in S$

Also, we have

$$
\begin{gathered}
\max \left\{\frac{x|\sin (2 x y)|}{2+y}, \frac{x^{2}}{2+x+y}, \frac{x|\sin (2 x+3 y)|}{2+y^{2}}, \frac{x}{2+y}, \frac{x}{2+|\sin (3 x+y)|}\right\} \\
\leq \frac{x}{2}=\varphi(x), \\
\sup _{y \in D}\left\{\frac{1}{3+x+y^{2}}+\max \left\{\frac{1}{5}\left|\cos \left(x+3 y^{2}\right)\right|, \frac{1}{4+x y}, \frac{1}{7+\left|\sin \left(3 x+y^{2}\right)\right|},\right.\right. \\
\left.\left.\frac{1}{6}|\cos (3 x+y+4)|\right\}\right\} \leq 1 .
\end{gathered}
$$

Thus it follows from Theorem 5.3 that the functional equation (71) possesses a solution $w \in B B(S)$. However the corresponding results in $[1,3,4,8,10$, $11,12,13,15,16,17]$ are not applicable for the functional equation (71). For this, we observe that $\forall(x, y) \in S \times D$

$$
|v(x, y)|=\frac{x^{3}}{1+\left|\sin \left(3 x y^{2}+4\right)\right|}>0,|r(x, y)|=\frac{1}{3+x+y^{2}}>0,
$$

and

$$
\left|t_{2}(x, y)\right|=\frac{x^{4}}{1+x+y^{2}}>0, \quad \forall(x, y) \in S \times D
$$

Also,

$$
|u(x, y)|=\frac{x^{4}}{1+x+y} \leq M|x|,
$$

does not holds for $(x, y)=(M+2,1)$, where $M$ is a positive constant. 
EXISTENCE AND UNIQUENESS OF SOLUTIONS FOR CERTAIN FUNCTIONAL EQUATIONS AND SYSTEM OF FUNCTIONAL EQUATIONS ARISING IN DYNAMIC PROGRAMMING

\section{Significance}

Dynamic programming has been applied to various type of problems in several different fields. The key elements associated with dynamic programming are stages, states, decisions, transformations, and returns. Dynamic programming occurs in multistage decision processes, i.e., it is an approach based upon the idea of imbedding any particular problem within a family of similar subproblems. This allows us to replace a multidimensional maximization problem by a corresponding problem of solving a system of recurrence relations involving functions of much smaller dimensions [1].

Bellman [1] proposed that there should be a formulation of the problem which preserves dimensionality and saves the researchers from becoming bogged down in the complexities of multidimensional analysis. Assume that we have a quantity $x$ which we divide into two nonnegative parts $y$ and $x-y$, obtaining from the first quantity $y$ a return of $g(y)$ and from the second a return of $h(x-y)$. If we wish to perform this division in such a way as to maximize the total return fare then taking preservation of one-dimensionality as a goal, Bellman [1] proceeded as follows: The maximum total return over an $N$-stage process depends only upon $N$ and the initial quantity $x$. Define the function,

$f_{N}(x)=$ the maximum return obtained from an $N$-stage process starting with an initial quantity $x \geq 0$, for $N=1,2, \ldots$.

Then we have

$$
f_{N}(x)=\operatorname{Max}_{(0<y<x)}\left[g(y)+h(x-y)+f_{N-1}(a(y)+b(x-y))\right], \quad N \geq 2 .
$$

If $N$ is large, it is reasonable to consider as an approximation to the $N$ stage process, the infinite process defined by the requirement that the process continues indefinitely. So, Bellman [1] suggested the following single equation for the above situation

$$
f(x)=\operatorname{Sup}_{(0 \leq y \leq x)}[g(y)+h(x-y)+f(a(y)+b(x-y))] .
$$

This approach was first applied to engineering control theory and then to other fields of applied mathematics, and later it became an important tool in economic theory. Unfortunately, many of the mathematical models of the universe, economic, physical, biological, or otherwise are large scale complex problems, the fastest computers and machines still require an appreciable time to determine the solution in this manner. Some of the difficulties in applying dynamic programming to real world problems are: inability of complete information of the constraints, and the computational time and feasibility of the solutions during the reduction of dimensionality of a complex problem of multistage decision making process into several interrelated subproblems of 
less dimensionality. For details of computational and approximate dynamic programming, see [18]. At the present time one of the crucial problems is to know that for the functional equations such as discussed in this work (which represent mathematical formulations of real world problems) solutions exist or not. Thus we conclude that our results will be useful to validate the existence of a unique solution, and of coincidence solutions.

Acknowledgement: This work is carried out under the project on Optimization and Reliability Modelling of Indian Statistical Institute, India.

\section{References}

[1] R. Bellman, Dynamic Programming, Princeton Univ. Press, Princeton, 1957.

[2] R. Bellman and E. S. Lee, Functional equations arising in dynamic programming, Aequationes Math. 17, 1-18 (1978).

[3] P. C. Bhakta and S. Mitra, Some existence theorems for functional equations arising in dynamic programming, J. Math. Anal. Appl. 98, 348-362 (1984).

[4] P. C. Bhakta and S. R. Choudhury, Some existence theorems for functional equations arising in dynamic programming II, J. Math. Anal. Appl. 131, 217-231 (1988).

[5] D. W. Boyd and J. S. W. Wong, On nonlinear contractions, Proc. Amer. Math. Soc. 20, 458-464 (1969).

[6] S. S. Chang, Some existence theorems of common and coincidence solutions for a class of functional equations arising in dynamic programming, Appl. Math. Mech., 12, 33-39 (1991).

[7] S. S. Chang and Y. H. Ma, Coupled fixed points for mixed monotone condensing operators and an existence theorem of the solutions for a class of functional equations arising in dynamic programming, J. Math. Anal. Appl., 160, 468-479 (1991).

[8] G. Jiang, S. M. Kang and Y. C. Kwun, Solvability and algorithms for functional equations originating from dynamic programming, Fixed Point Theory and Applications, Volume 2011, Article ID 701519, 30 pages, doi: 10.1155/2011/701519.

[9] Z. Liu, Existence theorems of solutions for certain classes of functional equations arising in dynamic programming, J. Math. Anal. Appl., 262, 529-553 (2001). 
EXISTENCE AND UNIQUENESS OF SOLUTIONS FOR CERTAIN FUNCTIONAL EQUATIONS AND SYSTEM OF FUNCTIONAL EQUATIONS ARISING IN DYNAMIC PROGRAMMING

[10] Z. Liu and S. M. Kang, Existence and uniqueness of solutions for two classes of functional equations arising in dynamic programming, Acta Mathematicae Applicatae Sinica. English Series, Vol. 23, No. 2, 195-208 (2007).

[11] Z. Liu and J. S. Ume, On properties of solutions for a class of functional equations arising in dynamic programming, Journal of Optimization Theory and Applications, 117, no. 3, 533-551 (2003).

[12] Z. Liu, R. P. Agarwal and S. M. Kang, On solvability of functional equations and system of functional equations arising in dynamic programming, J. Math. Anal. Appl. 297, 111-130 (2004).

[13] Z. Liu, S. M. Kang and J. S. Ume, Solvability and convergence of iterative algorithms for certain functional equations arising in dynamic programming, Optimization, 59, 887-916 (2010).

[14] Z. Liu, J. S. Ume and S. M. Kang, Some existence theorems for functional equations arising in dynamic programming, J. Korean Math. Soc. 43, 11 - 28 (2006).

[15] Z. Liu, J. S. Ume and S. M. Kang, Some existence theorems for functional equations and system of functional equations arising in dynamic programming, Taiwanese Journal of Mathematics, 14, 1517-1536 (2010).

[16] Z. Liu, Y. Xu, J. S. Ume and S .M. Kang, Solutions to two functional equations arising in dynamic programming, J. Comput. Appl. Math. 192, 251-269 (2006).

[17] H. K. Pathak and Deepmala, Existence and uniqueness of solutions of functional equations arising in dynamic programming, Appl. Math. Comp., 218 (2012) 7221-7230.

[18] W. B. Powell, Dynamic Programming: Solving the Curses of Dimensionality, John Wiley and Sons, Inc., Hoboken, New Jersey, 2007.

Deepmala,

SQC \& OR Unit,

Indian Statistical Institute,

203 B. T. Road, Kolkata, 700 108, India.

Email: dmrai23@gmail.com

Ravi P Agarwal,

Department of Mathematics,

Texas A\&M University-Kingsville,

Kingsville, TX 78363, USA.

Email: agarwal@tamuk.edu 